\title{
The burden of depression and anxiety among medical students in South Africa: A cross-sectional survey at the University of Cape Town
}

\author{
S van der Walt; ${ }^{1 \star}$ W S Mabaso; ${ }^{1 \star}$ E L Davids, ${ }^{2,3}$ BPsych, MA, PhD; P J de Vries, ${ }^{2}$ MB ChB, FRCPsych, PhD \\ ${ }^{1}$ Final-year medical student, Faculty of Health Sciences, University of Cape Town, South Africa \\ ${ }^{2}$ Adolescent Health Research Unit, Division of Child and Adolescent Psychiatry, Faculty of Health Sciences, University of Cape Town, South Africa \\ ${ }^{3}$ Cochrane South Africa, South African Medical Research Council, South Africa \\ * Joint first authors.
}

Corresponding authors: S van der Walt (svdwalt123@gmail.com), W S Mabaso (waks.mabaso@gmail.com)

\begin{abstract}
Background. Depressive and anxiety disorders occur at very high rates among medical students. For instance, an international review and meta-analysis estimated the overall pooled crude prevalence of depression or depressive symptoms at $27.2 \%$. However, South African (SA) data are very limited.

Objectives. To determine rates of depression and anxiety among medical students and to examine the associations with various sociodemographic variables (biological sex, gender identity, household income, ethnicity, and clinical v. pre-clinical students). We also examined whether the 'mini-semester' of 2017 resulting from the 2016 'Fees Must Fall' student protests was associated with increased depression/anxiety.

Methods. The study was a cross-sectional electronic survey conducted in the Faculty of Health Sciences, University of Cape Town, using an anonymous, self-administered online questionnaire. The questionnaire included basic sociodemographic questions, the Patient Health Questionnaire-9 and the Hospital Anxiety and Depression Scale.

Results. The sample consisted of 473 medical students, 333 (70.4\%) female and 140 (29.6\%) male. Based on ethnic self-identification, 165 (35.6\%) were black, $144(31.1 \%)$ white, $88(19 \%)$ coloured, $52(11.2 \%)$ Indian and 8 (1.7\%) Asian. There were 239 pre-clinical students (50.5\%) and 234 clinical students (49.5\%). Of the sample, $36.4 \%$ were above the cut-off for major depressive disorder and $45.9 \%$ for anxiety disorder. Reported rates of disorders diagnosed by a health professional were $25.0 \%$ for depressive disorder and $20.5 \%$ for anxiety disorder, and $28.1 \%$ of all students were receiving psychotropic medication. Female sex was associated with both depression (prevalence ratio (PR) 3.7; $p<0.001$ ) and anxiety diagnoses (PR 4.7; $p<0.001)$. None of the other sociodemographic characteristics showed significant associations. Interestingly, students who undertook the 2017 mini-semester showed an increased rate of depression $(\mathrm{PR} 2.1 ; p<0.05)$ and anxiety diagnoses (PR 2.1; $p<0.05$ ).

Conclusions. Nearly one in four students reported depression/anxiety diagnoses and were on psychotropic medication, significantly more than age-based expectations. Even more screened positive for risk of depression/anxiety. Our findings indicate that medical students, particularly females, are at a significantly increased risk of depression and anxiety disorders. Results suggest that the 2016 student protests may have had a direct impact on the mental state of students. Multidisciplinary efforts should be targeted at initiatives to strengthen mental wellbeing and institutional culture around mental health. These efforts may help build resilience in the next generation of health professionals in SA ahead of work in an overburdened health and health education system.
\end{abstract}

S Afr Med J 2020;110(1):69-76. https://doi.org/10.7196/SAMJ.2020.v110i1.14151

Mental health disorders are recognised by the World Health Organization (WHO) as significant contributors to morbidity and disability. Of these, depression currently ranks first. ${ }^{[1]}$ Systematic reviews have shown that medical students are disproportionately affected by this mental health burden, with high rates of depression or depressive symptoms (27.2\%), greater than their age-matched peers. ${ }^{[2,3]}$ In the 18 - 35-year age range, when students generally undergo training, the South African Stress and Mental Health (SASH) survey estimated the prevalence of major depressive disorder (MDD) at $8.9 \%$ and that of anxiety-related disorders at $14.6 \%{ }^{[4]}$ However, data on South African (SA) medical students are limited.

SA medical students undertake rigorous, extended training at an undergraduate level. Contextual factors that influence their experience may include financial difficulties, increased competition, language and cultural alienation, and under-preparedness for tertiary education. ${ }^{[5-7]}$ Although they are on privileged career paths relative to the majority of young people in the country, consideration should be given to the realities these paths entail at universities across the country. In addition, unique sociopolitical exposures accumulate and may be experienced as chronic stressors, increasing the risk of susceptibility to mental illness. ${ }^{[3,8,9]}$ These may include pressure on students to uplift their communities from poverty, regular exposure to societal inequity, victimisation and violence, threats to personal safety, delayed institutional transformation, and the impact of student protests on mental wellbeing. ${ }^{[10-13]}$ In January 2017, 2nd- and 3rd-year MB ChB students at the University of Cape Town underwent a contingency 'mini-semester' course as a result of postponement of classes and 2016 year-end examinations after curriculum time was lost to the 2016 'Fees Must Fall' student protests. The mini-semester comprised 6 weeks of teaching and assessments to make up for $\sim 2$ months of forfeited teaching and learning time, after which students could progress to their next scheduled academic year. 
Considering these contextual factors and the national discussion surrounding the mental wellbeing of junior doctors, clinicians and academics, it is prudent to investigate the profile of students who will fulfil these roles in the future ${ }^{[14]}$ Researchers from the University of KwaZulu-Natal investigated spirituality, depression and quality of life in 230 medical students. ${ }^{[15]}$ Utilising the Zung Self Rating Scale, they reported that $15.6 \%$ of students had scores suggestive of severe depression, with the highest scores found in students doing their 4th year of study. A second study, albeit using a non-validated questionnaire, examined 416 students at the University of Pretoria through years 1, 4, 5 and 6 . The researchers found that $14.9 \%$ of participants reported diagnoses of major depressive disorder. Respondents perceived increasing academic difficulty in the higher years of study. ${ }^{[16]}$ Both local studies suggested greater mental health symptom burdens in the clinical years of study. In international literature on medical student mental health, longitudinal studies showed a $13.5 \%$ median absolute increase in depressive symptoms after enrolment at medical school. ${ }^{[2]}$ Another multinational mental health study, which included 1st-year students from the University of Cape Town, reported MDD as the most common disorder, with a $21.2 \%$ lifetime prevalence. Generalised anxiety disorder was the second most common disorder, with a lifetime prevalence of $18.6 \%{ }^{[17]}$

\section{Objectives}

We set out to determine the rates of anxiety and depression symptoms, clinical diagnoses of depressive and anxiety disorders, and rates of psychotropic treatment among medical students at the University of Cape Town. In addition, we were interested in examining the associations between depressive/anxiety symptoms and disorders and a number of key sociodemographic factors. Given the potential contribution of the 'mini-semester' of 2017, we were also interested in examining the association between depression/ anxiety and participation in the mini-semester.

We hypothesised that medical students at the University of Cape Town would have comparable rates of depressive/anxiety symptoms to medical students at the universities of KwaZulu-Natal and Pretoria. We further predicted that a significant proportion of students would be on psychotropic medication, but at rates lower than those of actual diagnoses, suggesting undertreatment. We suspected that differences in the symptom burden of depression and anxiety would emerge between the pre-clinical (years 1 - 3) and clinical (years 4 - 6) years of study and hypothesised that the mini-semester negatively influenced student mental health, contributing to the burden of depressive/ anxiety symptoms and depression or anxiety diagnoses.

\section{Methods}

\section{Study setting and design}

A cross-sectional study at the Faculty of Health Sciences, University of Cape Town, SA, was undertaken from 5 to 19 October 2018. An anonymous, self-administered online questionnaire was developed using an electronic survey tool (Google Forms) and distributed to students through a variety of link-enabled platforms (email, student Facebook groups and student WhatsApp groups). Two gold-standard validated rating scales were utilised as measures for depressive symptoms (Patient Health Questionnaire-9 (PHQ-9) ${ }^{[18]}$ ) and anxiety symptoms (Hospital Anxiety and Depression Scale (HADS-A ${ }^{[19]}$ ). Questions from the WHO World Mental Health Composite International Diagnostic Interview (WMH-CIDI) were used for sociodemographic factors (Demographics long PAPI V7_1), ${ }^{[20]}$ and context-specific questions were developed by the authors, e.g. 'Did you undertake a mini-semester?.

\section{Participants}

All 1342 medical students enrolled in the 6-year MB ChB programme, including those undertaking their intercalated medical honours, were invited to participate. At the University of Cape Town, students are grouped as pre-clinical (years $1-3$ ) and clinical (years 4 - 6). Preclinical students mainly spend their time at the Faculty of Health Sciences campus and clinical students at the University of Cape Town teaching hospitals. To preserve validity of the PHQ-9 and HADS-A rating scales, students aged $<18$ years were excluded.

\section{Study definitions and instruments}

Major depressive disorder is a mental diagnosis that produces distinct episodes of persistent low mood and loss of interest in pleasurable activities for most days, lasting more than 2 weeks. Its symptoms affect mood, thoughts and the body, with a significant impact on daily function. In addition to the above major symptoms, others include sad, anxious or 'empty' feelings, feelings of worthlessness, guilt, irritability and poor sleep. ${ }^{[2]}$ These symptoms may vary in sequence, severity and duration, and do not immediately constitute diagnosis of a disorder, which requires a trained clinician. For the PHQ-9, the acceptable screening cut-off point is $\geq 10$ for risk of MDD according to the Diagnostic and Statistical Manual of Mental Disorders, 5 th edition. ${ }^{[21]}$

Anxiety disorders are also mental diagnoses, producing psychological and physical states of excessive uneasiness, fear or worry, causing significant distress, experienced over a variable 6-month period. Their symptoms are characterised by behavioural, emotional, physical and cognitive reactions to fear of a future threat that is out of proportion to its actual risk. ${ }^{[2]}$ These symptoms may also vary in sequence, severity and duration, and do not immediately constitute diagnosis of a disorder, which requires a trained clinician. ${ }^{[23]}$ For the HADS-A, an optimal balance of sensitivity and specificity for anxiety disorders $(0.80$ for both $)$ is established at the cut-off point of $\geq 8 .{ }^{[22,24,25]}$

Swartz et al. ${ }^{[26]}$ emphasise how cultural differences in upbringing inform the quality of experiences that SA university students endure. Differences attributed to languages and practices that are valued $v$. those that are not contribute to friction in learning. We therefore included self-identification ethnic classification to acknowledge the contestation of these differences as we sought to potentially discuss their roles as complex determinants. African students of black African descent were captured under 'black' and African students of mixed descent under 'coloured'.

\section{Data collection and procedure}

An online link directed students to the electronic consent form and information sheet. After consenting to participate, students were redirected to a secure page to complete the questionnaire. The study was advertised for 7 days with assistance from prominent student societies, class representatives and the faculty, before the link was closed.

\section{Contextual study bias}

In August 2018, the Dean of the Faculty of Health Sciences at the University of Cape Town, Prof. Bongani Mayosi, a leading academic and the nation's most prominent medical role model, ended his life as a result of major depression. ${ }^{[27]}$ This prompted a significant period of mourning, formally recognised by the faculty and informally experienced by the student body to varying degrees. Grief elicits depressive symptoms, and awareness of a suicide increases the risk of suicidality in people experiencing major depression. ${ }^{[28]}$ We therefore acknowledged this significant event as a potential confounder in our study. In order to mitigate the effect of the Dean's death, we 
let 9 weeks pass after his funeral (10 weeks from his death) before advertising the study.

\section{Ethics approval}

Research approval was granted by the University of Cape Town Faculty of Health Sciences Human Research Ethics Committee (ref. no. HREC 454/2018) after internal review of the protocol in the Department of Psychiatry and Mental Health. Institutional approval to access students was obtained from the Executive Director of the Department of Student Affairs.

\section{Data analysis}

All data were stored on password-protected computers and analysis was done using Stata 15 (StataCorp, USA). Basic descriptive analysis was done, and multivariate logistic regression analysis was used to determine associations between factors of interest and outcomes. A $95 \%$ confidence interval $(\mathrm{CI})$ was used and $p<0.05$ was considered significant.

\section{Results}

\section{Sample characteristics}

Four hundred and eighty-six responses were recorded. Thirteen respondents were excluded from analysis for not being currently enrolled in $\mathrm{MB} \mathrm{ChB}$, leaving 473 valid responses. For our multivariate analysis, a further 59 were excluded owing to missing fields. Our sample included an equal distribution among years of study, with $49.5 \%$ of students in their clinical years (years $4-6$ ) and the remainder in their pre-clinical years (years 1-3) or intercalated honours year (Table 1).

The median respondent age was 22 years. The majority of respondents were female $(70.4 \%)$, and black students made up the largest self-declared ethnic group (35.6\%), followed by white students (31.1\%). Cisgendered students represented $97.9 \%$ of the sample and $90.6 \%$ of respondents identified as heterosexual. Two hundred and fifteen students $(45.5 \%)$ had participated in the mini-semester in January 2017, $46.8 \%$ of students were receiving financial aid, and $60.5 \%$ of students' homes were $>500 \mathrm{~km}$ from campus. Most students reported a monthly family household income <ZAR40 000 (35.5\%), but a large proportion (34.0\%) did not know their monthly family household income. The majority of students (59.5\%) had a 60 - 79\% grade in their previous year, and $4.2 \%$ had completed previous degrees (Table 1).

\section{Depression and anxiety burden}

Table 2 shows the number of students above the cut-off (i.e. who screened in the 'at-risk' range) on the two validated rating scales as well as those who had been diagnosed with anxiety or depression by a health professional. On the rating scale measures, one in three students had symptom scores above the cut-off for depressive disorder, putting them in the at-risk range (36.4\% (95\% CI 32.0 40.9)), and nearly half had symptom scores above the cut-off for any anxiety disorder, putting them in the at-risk range (45.9\% (95\% CI 41.3 - 50.1)). In terms of clinical diagnoses of disorders, a quarter had been diagnosed with a depressive disorder, and one-fifth with an anxiety disorder (Table 2).

\section{Students receiving treatment}

Just over a quarter of all students $(28.1 \%$ (95\% CI 24.1 - 32.4) were receiving psychotropic medication; $16.1 \%$ (95\% CI 12.9 - 19.7) were on some form of psychotropic medication for depression, and $12.1 \%$ (95\% CI 9.3 - 15.3) for anxiety. Of students who had been diagnosed with depression, 58.5\% (95\% CI 49.0 - 67.5) were receiving some
Table 1. Demographic and socioeconomic background of MB ChB students in the study sample*

\begin{tabular}{|c|c|}
\hline Age at questionnaire (years), median (IQR) & $22(20-30)$ \\
\hline \multicolumn{2}{|l|}{ Year, $n(\%)(N=473)$} \\
\hline 1 & $76(16.1)$ \\
\hline 2 & $85(18.0)$ \\
\hline 3 & $73(15.4)$ \\
\hline 4 & $74(15.6)$ \\
\hline 5 & $95(20.1)$ \\
\hline 6 & $65(13.7)$ \\
\hline BSc Hons & $5(1.1)$ \\
\hline \multicolumn{2}{|l|}{ Sex, gender and sexual orientation, $n(\%)$} \\
\hline \multicolumn{2}{|l|}{ Sex assigned at birth $(N=473)$} \\
\hline Female & $333(70.4)$ \\
\hline Male & $140(29.6)$ \\
\hline \multicolumn{2}{|l|}{ Gender identity $(N=470)$} \\
\hline Cisgender, woman & $323(68.7)$ \\
\hline Cisgender, man & $137(29.2)$ \\
\hline Non-binary & $5(1.1)$ \\
\hline Transgender, woman & $4(0.9)$ \\
\hline Transgender, man & 0 \\
\hline \multicolumn{2}{|l|}{ Sexual orientation $(N=466)$} \\
\hline Heterosexual & $422(90.6)$ \\
\hline Bisexual & $23(4.9)$ \\
\hline Gay & $9(1.9)$ \\
\hline Queer & $7(1.5)$ \\
\hline Lesbian & $2(0.4)$ \\
\hline Mostly heterosexual & $1(0.2)$ \\
\hline \multicolumn{2}{|l|}{ Race, $n(\%)(N=463)$} \\
\hline Black & $165(35.6)$ \\
\hline White & $144(31.1)$ \\
\hline Coloured & $88(19)$ \\
\hline Indian & $52(11.2)$ \\
\hline Asian & $8(1.7)$ \\
\hline Other & $6(1.3)$ \\
\hline \multicolumn{2}{|l|}{ Underwent mini-semester, $n(\%)(N=473)$} \\
\hline Yes & $215(45.5)$ \\
\hline No & $258(54.5)$ \\
\hline \multicolumn{2}{|l|}{ Uses financial aid, $n(\%)(N=464)$} \\
\hline Yes & $217(46.8)$ \\
\hline No & $247(53.2)$ \\
\hline \multicolumn{2}{|l|}{ Distance from home $(\mathrm{km}), n(\%)(N=456)$} \\
\hline$>500$ & $276(60.5)$ \\
\hline$<500$ & $180(39.5)$ \\
\hline \multicolumn{2}{|l|}{ Previous degree, $n(\%)(N=475)$} \\
\hline Yes & $20(4.2)$ \\
\hline No & $455(95.8)$ \\
\hline \multicolumn{2}{|l|}{ Previous year's marks (\%), $n(\%)(N=462)$} \\
\hline $80-100$ & $106(22.9)$ \\
\hline $60-79$ & $275(59.5)$ \\
\hline $40-59$ & $78(16.9)$ \\
\hline$<40$ & $3(0.7)$ \\
\hline \multicolumn{2}{|l|}{$\begin{array}{l}\text { Family household income per month after tax } \\
\text { (ZAR), } n(\%)(N=473)\end{array}$} \\
\hline$<40000$ & $168(35.5)$ \\
\hline $40000-60000$ & $46(9.7)$ \\
\hline$>60000$ & $98(20.7)$ \\
\hline Unknown & $161(34.0)$ \\
\hline
\end{tabular}


Table 2. Rates of depression and anxiety symptoms above the cut-off suggesting risk of depression and anxiety disorders, and clinical diagnoses of depression and anxiety

\begin{tabular}{|c|c|c|c|c|c|c|}
\hline & \multicolumn{2}{|r|}{ At risk ${ }^{*}$} & \multicolumn{2}{|c|}{ Clinical diagnosis } & \multicolumn{2}{|r|}{ Using psychotropic medication } \\
\hline & $n$ & $\%(95 \% \mathrm{CI})$ & $n$ & $\%(95 \% \mathrm{CI})$ & $n$ & $\%(95 \% \mathrm{CI})$ \\
\hline Depression & 172 & $36.4(32.0-40.9)$ & 118 & $25.0(21.2-29.2)$ & 76 & $16.1(12.9-19.7)$ \\
\hline Anxiety disorder (any) & 217 & $45.9(41.3-50.1)$ & 97 & $20.5(17.0-24.5)$ & 57 & $12.1(9.3-15.3)$ \\
\hline
\end{tabular}

form of treatment (psychotherapy, pharmacotherapy, other), as were 66.0\% (95\% CI 55.7 - 75.3) of students who had been diagnosed with an anxiety disorder (not shown in table).

\section{Associations with sociodemographic factors}

The following sociodemographic associations were made with depressive symptoms that scored above the cut-off for being at risk of depressive disorder. Compared with males, females had a significantly higher PR for risk of depressive disorder (PR 1.9 (95\% CI 1.2 - 3.2); $p<0.01)$. Clinical students and heterosexual students showed decreased associations with symptom scores indicating a risk of depressive disorder (PR 0.6 (95\% CI $0.4-1.0$ ); $p<0.05$ and PR 0.5 (95\% CI $0.2-1.0$ ); $p<0.05$, respectively) (Table 3 ).

Among students with a diagnosis of depressive disorder, the PR was also significantly higher for females (PR 3.7 (95\% CI 2.0 - 6.8); $p<0.001)$ compared with males. Similarly, we observed an increased PR for depressive disorder in mini-semester students (PR 2.1 (95\% CI 1.3 - 3.4); $p<0.005)$ and students who had completed a previous degree (PR 3.0 (95\% CI 1.1 - 8.7); $p<0.005$ ) (Table 3).

With regard to anxiety symptom scores above the cut-off for being at risk of anxiety disorders, females had a significantly higher PR than males (PR 1.6 (95\% CI 1.0 - 2.6); $p<0.05)$. Females were also significantly more likely to have an anxiety disorder diagnosis than males (PR 4.7 (95\% CI 2.2 - 9.8); $p<0.001$ ) as were mini-semester students compared with students who had not done the minisemester (PR 2.1 (95\% CI 1.2 - 3.5); $p<0.05)$ (Table 4).

\section{Discussion}

The present study aimed to determine rates of depressive and anxiety symptoms and clinical disorders among medical students, and their associations with a range of sociodemographic and sociopolitical factors. We predicted that medical students at the University of Cape Town would have comparable rates of depressive/anxiety symptoms to medical students at other SA universities and that a large proportion of medical students would be on psychotropic medication for depressive/anxiety symptoms, but at rates lower than suggested by clinical diagnoses. We also predicted differences in the depressive/anxiety symptom burden between the pre-clinical (years 1 - 3) and clinical students (years 4 - 6), and that undergoing the 2017 mini-semester following the 2016 Fees Must Fall protests contributed to the burden of depressive/anxiety symptoms and diagnoses.

Over a third of students had symptoms above the cut-off for depressive disorder (36.4\%) and a quarter had a clinical diagnosis of depression (25.0\%). Just under half of the students had symptoms indicating that they were at risk of anxiety disorders (45.9\%), and one-fifth had an anxiety disorder diagnosis (20.5\%). The profile of depression and anxiety symptoms assessed by the PHQ-9 and HADS-A scales was therefore very high, and rates of depression and anxiety diagnoses in medical students were significantly higher than rates for the age-matched general population $(8.9 \%$ and $14.6 \%){ }^{[4]}$
The depressive symptom burden in medical students at the University of Cape Town in this study was higher than local findings from the University of KwaZulu-Natal (15.6\%), ${ }^{[15]}$ the University of the Free State $(26.6 \%)^{[29]}$ and the University of Pretoria (14.9\%). ${ }^{[16]}$ We acknowledge that it is unclear whether these findings represent true differences or may be attributable to differences in measurement tools and/or the impact of the death of the Dean of the Faculty of Health Sciences at the University of Cape Town. Nevertheless, our findings provide local evidence of the significant burden of depressive and anxiety symptoms and disorders in medical students. We showed that a significant proportion $(28.1 \%)$ of medical students were on psychotropic medication, but, as predicted, showed that only $58.5 \%$ of students with clinical depression and $66.0 \%$ with anxiety diagnoses were receiving some form of treatment, underlining the treatment gap of these disorders among medical students.

A study of first-year university students conducted by Auerbach et al. ${ }^{[17]}$ in eight countries, including SA, showed that $35 \%$ of students reported at least one lifetime mental disorder. They found that major depressive disorder was the most common of all the disorders assessed, with a lifetime prevalence of $21.2 \%$, and that the lifetime prevalence of generalised anxiety disorder was $18.6 \%$. These findings were comparable to our rates of $25 \%$ and $20.5 \%$, respectively.

Apart from examination of rates and treatment of depression and anxiety, we were also interested in sociodemographic and sociopolitical correlates with these disorders. Previous longitudinal studies suggested that, after a peak in the earlier years of medical school, depression symptom rates undergo a gradual decline in the later years. ${ }^{[30,31]}$ We showed that students in their clinical years had a decreased association with being at risk of a depressive disorder, suggesting support of similar previous findings. ${ }^{[15,16]}$ However, the findings for clinical diagnoses of depressive disorder and anxiety did not support these predictions. Interestingly, however, we did confirm that students who undertook the 2017 mini-semester had an increased likelihood of a clinical diagnosis of depressive or anxiety disorder. To our knowledge, this is the first evidence to indicate such sociopolitical factors as potential risk markers for mood disorders in SA medical students.

Students who scored higher marks in the previous year appeared to be protected against risk and diagnosis of depression in a doseresponse fashion, but no similar effect was observed for anxiety. It is unclear whether higher marks protected against depression or not experiencing depression allowed for better academic performance. Considering that some individuals with depression or at risk may have exhibited high-functioning phenomena, the exact meanings behind these findings become more difficult to elucidate.

Increased associations between female sex and both symptoms indicating risk of depressive/anxiety disorder and clinical diagnosis of depressive/anxiety disorder were confirmed. Our study showed a decreased association between heterosexual students and symptoms 
Table 3. Multivariate logistic regression analysis for depression $(N=414)$

\begin{tabular}{|c|c|c|c|c|}
\hline & \multicolumn{2}{|c|}{ At risk of depression } & \multicolumn{2}{|c|}{ Depression diagnosis } \\
\hline & aOR $(95 \% \mathrm{CI})$ & $p$-value & aOR $(95 \% \mathrm{CI})$ & $p$-value \\
\hline \multicolumn{5}{|l|}{ Year } \\
\hline Clinical & $0.6(0.4-1.0)$ & $<0.05^{*}$ & $1.2(0.7-2.0)$ & 0.6 \\
\hline Pre-clinical & & & 1 & \\
\hline \multicolumn{5}{|c|}{ Sex, gender and sexual orientation } \\
\hline \multicolumn{5}{|c|}{ Sex assigned at birth } \\
\hline Female & $1.9(1.2-3.2)$ & $<0.01^{*}$ & $3.7(2.0-6.8)$ & $<0.001^{*}$ \\
\hline Male & & & 1 & \\
\hline \multicolumn{5}{|l|}{ Gender identity } \\
\hline Cisgender & $0.8(0.2-3.5)$ & 0.7 & $1.0(0.2-5.0)$ & 1.0 \\
\hline Transgender & & & 1 & \\
\hline \multicolumn{5}{|l|}{ Sexual orientation } \\
\hline Heterosexual & $0.5(0.2-1.0)$ & $<0.05^{*}$ & $0.6(0.3-1.3)$ & 0.2 \\
\hline Not heterosexual & & & 1 & \\
\hline \multicolumn{5}{|l|}{ Race } \\
\hline Black & $1.0(0.2-5.6)$ & 1.0 & $3.1(0.3-31.2)$ & 0.3 \\
\hline White & $0.9(0.2-4.7)$ & 0.9 & $2.9(0.3-28.4)$ & 0.4 \\
\hline Coloured & $0.7(0.1-4.0)$ & 0.7 & $2.1(0.2-23.1)$ & 0.5 \\
\hline Indian & $1.1(0.2-6.6)$ & 0.9 & $1.8(0.2-19.7)$ & 0.6 \\
\hline Other & $1.7(0.1-21.5)$ & 0.7 & $3.8(0.2-71.2)$ & 0.4 \\
\hline Asian & & & 1 & \\
\hline \multicolumn{5}{|l|}{ Mini-semester } \\
\hline Yes & $1.3(0.8-2.0)$ & 0.3 & $2.1(1.3-3.4)$ & $<0.005^{*}$ \\
\hline No & & & 1 & \\
\hline \multicolumn{5}{|l|}{ Financial aid } \\
\hline Yes & $1.0(0.6-1.7)$ & 1.0 & $0.7(0.4-1.2)$ & 0.2 \\
\hline No & & & 1 & \\
\hline \multicolumn{5}{|c|}{ Distance from home $(\mathrm{km})$} \\
\hline$>500$ & $1.1(0.7-1.8)$ & 0.7 & $1.1(0.6-1.9)$ & 0.7 \\
\hline$<500$ & & & 1 & \\
\hline \multicolumn{5}{|l|}{ Previous degree } \\
\hline Yes & $0.9(0.3-2.7)$ & 0.8 & $3.0(1.1-8.7)$ & $<0.05^{*}$ \\
\hline No & & & 1 & \\
\hline \multicolumn{5}{|l|}{ Previous year's mark (\%) } \\
\hline $80-100$ & $0.4(0.2-0.8)$ & $<0.01^{*}$ & $0.3(0.2-0.8)$ & $<0.05^{*}$ \\
\hline $60-79$ & $0.5(0.3-0.9)$ & $<0.05^{*}$ & $0.5(0.3-1.1)$ & 0.07 \\
\hline $40-59$ & & & 1 & \\
\hline$<40$ & - & - & $2.6(0.2-37.2)$ & 0.5 \\
\hline \multicolumn{5}{|c|}{ Family household income per month (ZAR) } \\
\hline$>60000$ & $1.4(0.6-3.3)$ & 0.5 & $0.7(0.3-1.6)$ & 0.4 \\
\hline $40000-60000$ & & & 1 & \\
\hline$<40000$ & $1.7(0.7-4.0)$ & 0.2 & $0.9(0.4-2.3)$ & 0.9 \\
\hline Unknown & $1.3(0.6-3.1)$ & 0.5 & $0.9(0.4-2.1)$ & 0.8 \\
\hline
\end{tabular}

indicating risk of depressive disorder. The literature indicates that poorer mental health outcomes in non-heterosexual individuals are linked to direct and perceived societal stressors, showing two- to three-fold elevated odds of lifetime mental health disorders. ${ }^{[17,32,33]}$ Other sociodemographic factors such as ethnicity, receiving financial aid, monthly family household income and distance from home did not show significant associations.

In SA, financial difficulty is recognised as the most difficult stressor for students to control. ${ }^{[26]}$ Students who do not qualify for financial aid but whose families require it fall into what is called the 'missing middle'. Students in this 'missing middle' could have been at greater risk for mental health associations than students who qualify for financial assistance and students who do not require it, an important consideration for future studies. We did not investigate the role of religion or spirituality, but the University of KwaZulu-Natal study on depression and spirituality highlighted its protective role on mental health. ${ }^{[15]}$

Students who underwent the mini-semester were significantly more likely to have a diagnosis of depression and/or anxiety than those who did not, an important finding in the context of the SA 
Table 4. Multivariate logistic regression analysis for any anxiety disorder $(N=414)$

\begin{tabular}{|c|c|c|c|c|}
\hline & \multicolumn{2}{|c|}{ At risk of anxiety } & \multicolumn{2}{|c|}{ Anxiety diagnosis } \\
\hline & aOR $(95 \% \mathrm{CI})$ & $p$-value & aOR $(95 \% \mathrm{CI})$ & $p$-value \\
\hline \multicolumn{5}{|l|}{ Year } \\
\hline Clinical & $0.7(0.5-1.1)$ & 0.1 & $0.7(0.4-1.2)$ & 0.2 \\
\hline Pre-clinical & & & 1 & \\
\hline \multicolumn{5}{|c|}{ Sex, gender and sexual orientation } \\
\hline \multicolumn{5}{|c|}{ Sex assigned at birth } \\
\hline Female & $1.6(1.0-2.6)$ & $<0.05^{*}$ & $4.7(2.2-9.8)$ & $<0.001^{\star}$ \\
\hline Male & & & 1 & \\
\hline \multicolumn{5}{|l|}{ Gender identity } \\
\hline Cisgender & $1.2(0.3-5.0)$ & 0.8 & $0.3(0.1-1.5)$ & 0.1 \\
\hline Transgender & & & 1 & \\
\hline \multicolumn{5}{|l|}{ Sexual orientation } \\
\hline Heterosexual & $0.7(0.4-1.5)$ & 0.4 & $0.6(0.2-1.3)$ & 0.2 \\
\hline Not heterosexual & & & 1 & \\
\hline \multicolumn{5}{|l|}{ Race } \\
\hline Black & $0.9(0.2-4.2)$ & 0.9 & $0.6(0.1-4.0)$ & 0.6 \\
\hline White & $1.2(0.3-5.5)$ & 0.8 & $0.7(0.1-4.7)$ & 0.7 \\
\hline Coloured & $1.5(0.3-7.2)$ & 0.6 & $0.5(0.1-3.8)$ & 0.6 \\
\hline Indian & $1.7(0.3-8.6)$ & 0.5 & $0.5(0.1-3.5)$ & 0.5 \\
\hline Asian & & & 1 & \\
\hline Other & $0.8(0.1-8.1)$ & 0.8 & $0.9(0.1-12.3)$ & 0.7 \\
\hline \multicolumn{5}{|l|}{ Mini-semester } \\
\hline Yes & $1.5(1.0-2.2)$ & 0.06 & $2.1(1.2-3.5)$ & $<0.05^{*}$ \\
\hline No & & & 1 & \\
\hline \multicolumn{5}{|l|}{ Financial aid } \\
\hline Yes & $0.9(0.6-1.5)$ & 0.8 & $0.9(0.5-1.7)$ & 0.8 \\
\hline No & & & 1 & \\
\hline \multicolumn{5}{|l|}{ Distance from home $(\mathrm{km})$} \\
\hline$>500$ & $1.3(0.8-2.0)$ & 0.3 & $0.8(0.4-1.4)$ & 0.5 \\
\hline$<500$ & & & 1 & \\
\hline \multicolumn{5}{|l|}{ Previous degree } \\
\hline Yes & $1.0(0.4-2.6)$ & 1.0 & $2.0(0.6-6.3)$ & 0.2 \\
\hline No & & & 1 & \\
\hline \multicolumn{5}{|l|}{ Previous year's mark (\%) } \\
\hline $80-100$ & $0.8(0.4-1.5)$ & 0.5 & $0.7(0.3-1.8)$ & 0.4 \\
\hline $60-79$ & $0.9(0.5-1.6)$ & 0.7 & $1.6(0.7-3.5)$ & 0.2 \\
\hline $40-59$ & & & 1 & \\
\hline$<40$ & $1.5(0.1-18.7)$ & 0.7 & $7.4(0.5$ - 99.6) & 0.1 \\
\hline \multicolumn{5}{|c|}{ Family household income per month (ZAR) } \\
\hline$>60000$ & $0.6(0.3-1.3)$ & 0.2 & $0.6(0.2-1.5)$ & 0.3 \\
\hline $40000-60000$ & & & 1 & \\
\hline$<40000$ & $0.8(0.4-1.7)$ & 0.6 & $0.6(0.2-1.4)$ & 0.2 \\
\hline Unknown & $0.9(0.4-1.8)$ & 0.7 & $0.6(0.3-1.4)$ & 0.3 \\
\hline
\end{tabular}

narrative around student protests. ${ }^{[7,26]}$ There are reflections to be made by all concerned (students, staff, parents and the general public) regarding the psychological sequelae of prolonged protests required to effect change. Swartz et al. ${ }^{[26]}$ described the period of protests preceding the mini-semester as months of tension where praxis and access to tertiary education were contested. ${ }^{[26]}$ Many students made significant personal, social and academic sacrifices to challenge the longstanding shortcomings in tertiary funding and institutional culture. Our findings on the high burden of mental health disorders in university medical students encourages reflection on these sacrifices in an evolving tertiary education landscape.
Many of the sociodemographic factors that we expected to show independent associations with depression and anxiety symptoms or disorders did not (monthly household income, distance from home and ethnicity). However, these factors may intersect towards susceptibility: compounded over time they may undermine mental health by eroding students' coping mechanisms as a function of their chronicity, regardless of initial adaptive coping responses. ${ }^{[8,26]}$ In 2018, 19 students took long leave of absence from the University of Cape Town's Faculty of Health Sciences due to mental illness. No students in the faculty were reported to have ended their lives as a result of mental illness, but the Dean's suicide emphasised major depression's worst outcome. 
The recent longitudinal qualitative study of eight SA universities by Swartz et al. ${ }^{[26]}$ provided simple recommendations for mental health in tertiary institutions: reduce the stigma attached to mental health issues and publicise available counselling and medical services, train lecturers/clinical educators to deal with students' mental health issues, improve their own competencies as first points of referral, arrange mental health outreach, and incentivise healthy behaviours and resilience strengthening during stressful periods. Mixed emerging evidence on resilience supports actions such as making improvements to the student environment, mentorship between students and their qualified seniors, interpersonal support among students that advances the narrative of peers on a privileged path, and prioritising wellbeing throughout undergraduate study by supporting physical exercise, a healthy diet, self-awareness and spirituality. ${ }^{[34]}$ The University of Cape Town has championed against stigma by drafting a mental health policy and continues to increase visibility and access to counselling services, setting the tone for students and clinicians alike to address the institutional culture in medicine and improve mental wellbeing. ${ }^{[2,35]}$

\section{Study limitations}

We acknowledge a number of potential limitations to our study. First, cross-sectional studies cannot be interpreted to imply causality, but our findings raise important risk markers to consider in future studies. Second, we acknowledge a number of potential biases in our methodology. We have already raised the contextual bias around the death of Prof. Mayosi as a factor that may have led to increased rates of depressive/anxiety symptoms at the time of our study as well as having an influence on response rate. Conversely, we acknowledge that the absence of students who deregistered due to poor mental health, long leave of absence or financial/academic exclusion may have reduced rates of anxiety/depression in our sample. Survey methodologies are always at risk of reduced participation by students with high levels of anxiety/depressive symptoms. Notwithstanding these limitations, our findings provided strong local data related to depressive and anxiety symptoms and disorders among medical students in an SA setting.

\section{Conclusions}

The present study showed a high burden of depression and anxiety in medical students, but suggested undertreatment of both depressive and anxiety disorders. It confirmed the well-known association between female sex and depressive/anxiety disorders. Very interestingly, the study provided the first-ever evidence of uniquely SA sociopolitical factors (the 2017 mini-semester) and their association with depression/anxiety. This is the largest study on depression and anxiety in SA medical students to date. However, we believe that further research at other faculties as well as longitudinal studies following students throughout their studies and into their careers as junior doctors could further strengthen our understanding. To improve on the current burden, we recommend expansion of mental health services at the Faculty of Health Sciences. In addition, we recommend that health sciences faculties consider initiatives for faculty-wide mental wellbeing. We propose that an improvement in the culture and values of medical education towards positive role modelling, prioritising wellbeing, strengthening resilience and maintaining mental health could translate into an environment that prepares students for making a meaningful impact on others while attaining health, wellbeing and success in their own lives. With a focus on support, role modelling and mentorship by faculty, clinicians and students we can stimulate healthy emotional growth, and the early prevention and management of our mental health burden.

\section{Declaration. None.}

Acknowledgements. The authors thank the Faculty of Health Sciences at the University of Cape Town and peers in the student community for prioritising and encouraging this research. We also thank Suniti Sinha and Josh Fieggen for their help in data analysis and Stephanie Roche for help with the questionnaire.

Author contributions. SvdW and WSM conceptualised the research questions. All authors contributed to protocol development. SvdW and WSM performed data collection. SvdW performed statistical data analysis and WSM drafted the manuscript. ELD and PJdV contributed as supervisors of the research, guiding each stage of research development. All authors contributed to the interpretation of the analysis and critical revision of the manuscript, and approved the final version of the manuscript. SvdW and WSM contributed equally to this study and should be regarded as joint first authors.

Funding. None.

Conflicts of interest. None.

Oral presentation. The key findings of this article were presented at the 20th Conference of the South African Association of Child and Adolescent Psychiatry and Allied Professions, Johannesburg, 23 - 25 May 2019.

1. World Health Organization. Depression. Geneva: WHO, 2018. https://www.who.int/news-room/factsheets/detail/depression (accessed 2 February 2018).

2. Rotenstein LS, Ramos MA, Torre M, et al. Prevalence of depression, depressive symptoms, and suicidal ideation among medical students. JAMA 2016;316(21):2214-2236. https://doi.org/10.1001/ suicidal ideation
jama.2016.17324

3. Jeong Y, Kim JY, Ryu JS, Lee K, Ha EH, Park H. The associations between social support, healthJeong Y, Kim JY, Ryu JS, Lee K, Ha EH, Park H. The associations between social support, health-
related behaviors, socioeconomic status and depression in medical students. Epidemiol Health related behaviors, socioeconomic status and depression
2010;32:e2010009. https://doi.org/10.4178/epih/e2010009

4. Herman AA, Stein DJ, Seedat S, Heeringa SG, Moomal H, Williams DR. The South African Stress and Health (SASH) study: 12-month and lifetime prevalence of common mental disorders. S Afr Med J 2009;99(5):339-344.

5. Crowe T. Accountability only way to solve the problems confronting UCT. Cape Town: Independent Media, 2016. https://www.iol.co.za/capetimes/news/accountability-only-way-to-solve-the-problemsconfronting-uct-2080868 (accessed 7 March 2018).

6. Shepherd J. Speak out if you dare. The Guardian, 2009. https://www.theguardian.com/education/2009/ feb/17/national-union-of-students (accessed 7 March 2018)

Various contributors. A commentary on matters of the Faculty of Health Sciences at the University of Cape Town. Student support and basic support structures. Faculty of Health Sciences, University of Cape Town, 2017

8. Wheaton B, Montazer S. Stressors, stress, and distress. In: Scheid TL, Brown TN, eds. A Handbook for the Study of Mental Health: Social Contexts, Theories and Systems. 2nd ed. New York: Cambridge University Press, 2010:171-199. https://doi.org/10.1017/CBO9780511984945

9. Wahed WYA, Hassan SK. Prevalence and associated factors of stress, anxiety and depression among medical Fayoum University students. Alexandria J Med 2017;53(1):77-84. https://doi.org/10.1016/j. ajme.2016.01.005

10. Karim AA. Black tax. Cape Town: Varsity Newspaper, 2016:5;75. https://issuu.com/varsitynewspaper/ docs/merged_2_ (accessed 6 February 2018).

1. Karim AA. Mental health is no joke. Cape Town: Varsity Newspaper, 2016:4;75. https://issuu.com/ varsitynewspaper/docs/merged_1_319175a6496aba (accessed 6 February 2018).

12. Karim AA. Decolonising the university. Cape Town: Varsity Newspaper, 2016:3;75. https://issuu.com/ varsitynewspaper/docs/merged (accessed 6 February 2018).

13. Demands made by the students of the Faculty of Health Sciences. Faculty of Health Sciences, University of Cape Town, 2016:1-3.

14. Peterson C. Young doctors dangerously overworked. Cape Town: Independent Media, 2016. https:// www.iol.co.za/news/south-africa/western-cape/young-doctors-dangerously-overworked-2034705 www.iol.co.za/news/sout
(accessed 5 January 2018)

15. Pillay N, Ramlall S, Burns JK. Spirituality, depression and quality of life in medical students in KwaZulu-Natal. S Afr J Psychiatry 2016;22(1):1-6. https://doi.org/10.4102/sajpsychiatry.v22i1.731

16. Van Niekerk L, Viljoen A, Rischbieter P, et al. Subjective experience of depressed mood among medical students at the University of Pretoria. S Afr J Psychiatry 2008;14(1):27-31. https://doi.org/10.4102/ sajpsychiatry.v14i1.42

17. Auerbach RP, Mortier P, Bruffaerts R, et al. WHO World Mental Health Surveys International College Student Project: Prevalence and distribution of mental disorders. J Abnorm Psychol 2018;127(7):623638. https://doi.org/10.1037/abn0000362

8. Kroenke K, Spitzer RL, Williams JBW. Patient Health Questionnaire-9. PsycTESTS Dataset. American Psychiatric Association, 1999. https://doi.org/10.1037/t06165-000

9. Zigmond AS, Snaith RP. Hospital Anxiety and Depression Scale. PsycTESTS Dataset. American Psychiatric Association, 1983. https://doi.org/10.1111/j.1600-0447.1983.tb09716.x

20. Nelson C. The Composite International Diagnostic Interview. Geneva: World Health Organization, 1999;77(7):614.

21. Depressive Disorders. Diagnostic and Statistical Manual of Mental Disorders. 5th ed. American Psychiatric Association, 2019. https://dsm.psychiatryonline.org/doi/abs/10.1176/appi. American Psychiatric Association, 2019. https://
books.9780890425596.dsm04 (accessed 2 April 2019).

22. Ali G-C, Ryan G, de Silva MJ. Validated screening tools for common mental disorders in low and middle income countries: A systematic review. PLoS One 2016;11(6).e0156939. https://doi org/10.1371/journal.pone. 0156939

23. Stein DJ. Anxiety and Related Disorders in DSM-5. Anxiety Disorders. Oxford University Press, 2015:3-16. https://doi.org/10.1093/med/9780199395125.003.0001

24. Erschens R, Herrmann-Werner A, Bugaj T, Nikendei C, Zipfel S, Junne F. Methodological aspects of international research on the burden of anxiety and depression in medical students. Mental Health Prev 2016;4(1):31-35. https://doi.org/10.1016/j.mhp.2016.01.003 
25. Bjelland I, Dahl AA, Haug TT, et al. The validity of the Hospital Anxiety and Depression Scale: An updated literature review. J Psychosom Res 2002;52(2):69-77. https://doi.org/10.1016/S0022 3999(01)00296-3

26. Swartz S, Mahali A, Moletsane R, et al. Studying while Black: Race, Education and Emancipation in South African universities. Cape Town: HSRC Press, 2018

27. Khumalo Mayosi N. Yes, we did fail Bongani Mayosi. S Afr Med J 2018;108(9):697-698. https://doi org/10.7196/SAMJ.2018.v108i9.13609

28. Wakefield JC, Schmitz MF. The measurement of mental disorder. In: Scheid TL, Brown TN, eds A Handbook for the Study of Mental Health: Social Contexts, Theories and Systems. 2nd ed. New York: Cambridge University Press, 2010:20-45. https://doi.org/10.1017/CBO9780511984945.005

29. Van Zyl PM, Joubert G, Bowen E, et al. Depression, anxiety, stress and substance use in medical students in a 5-year curriculum. Afr J Health Prof Educ 2017;9(2):67-72. https://doi.org/10.7196 AJHPE.2017.v9i2.705

30. Roh M-S, Jeon HJ, Kim H, et al. The prevalence and impact of depression among medical students: A nationwide cross-sectional study in South Korea. Acad Med 2010;85(8):1384-1390. https.//dol. org/10.1097/ACM.0b013e3181df5e 43
31. Dyrbye LN, Thomas MR, Shanafelt TD. Systematic review of depression, anxiety, and other indicators of psychological distress among U.S. and Canadian medical students. Acad Med 2006;81(4):354-373. https://doi.org/10.1097/00001888-200604000-00009

32. King M, Semlyen J, Tai SS, et al. A systematic review of mental disorder, suicide, and deliberate self harm in lesbian, gay and bisexual people. BMC Psychiatry 2008;8(1):70. https://doi.org/10.1186/1471244X-8-70

. Müller A. Beyond 'invisibility': Queer intelligibility and symbolic annihilation in healthcare. Cult Health Sex 2018;20(1):14-27. https://doi.org/10.1080/13691058.2017.1322715

34. Ludwig AB, Burton W, Weingarten J, Milan F, Myers DC, Kligler B. Depression and stress amongst undergraduate medical students. BMC Med Educ 2015;15(1):141. https://doi.org/10.1186/s12909015-0425-z

35. Helmich E, Bolhuis S, Koopmans R. Medical students and depression. JAMA 2011;305(1):38-39. https://doi.org/10.1001/jama.2010.1886

Accepted 15 July 2019 